Supporting Information

\title{
Ultrasound-Switchable Nanozyme Augments Sonodynamic Therapy against Multidrug- Resistant Bacterial Infection
}

Duo Sun, ${ }^{1, \#}$ Xin Pang, ${ }^{2, \#}$ Yi Cheng, ${ }^{2, \#}$ Jiang Ming, ${ }^{1}$ Sijin Xiang, ${ }^{1}$ Chang Zhang, ${ }^{2}$ Peng Lv, ${ }^{2}$ Chengchao Chu, ${ }^{2}$ Xiaolan Chen, ${ }^{1, *}$ Gang Liu, ${ }^{2}, *$ and Nanfeng Zheng ${ }^{1, *}$

${ }^{1}$ State Key Laboratory for Physical Chemistry of Solid Surfaces, Collaborative Innovation Center of Chemistry for Energy Materials and Engineering, Research Center for Nano-Preparation Technology of Fujian Province, College of Chemistry and Chemical Engineering, Xiamen University, Xiamen 361005, China.

*E-mail: chenxl@xmu.edu.cn; nfzheng@xmu.edu.cn

${ }^{2}$ State Key Laboratory of Molecular Vaccinology and Molecular Diagnostics \& Center for Molecular Imaging and Translational Medicine, School of Public Health, Xiamen University, Xiamen 361102, China.

*E-mail: gangliu.cmitm@xmu.edu.cn

\# These authors contributed equally to this study. 


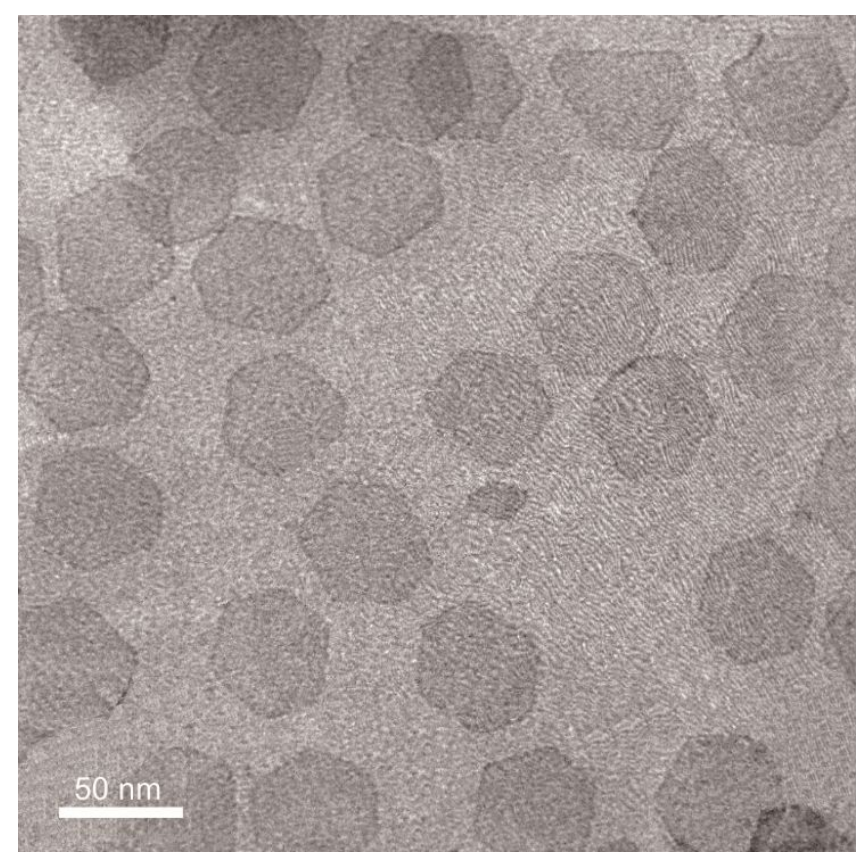

Figure S1. TEM image of Pd NSs.

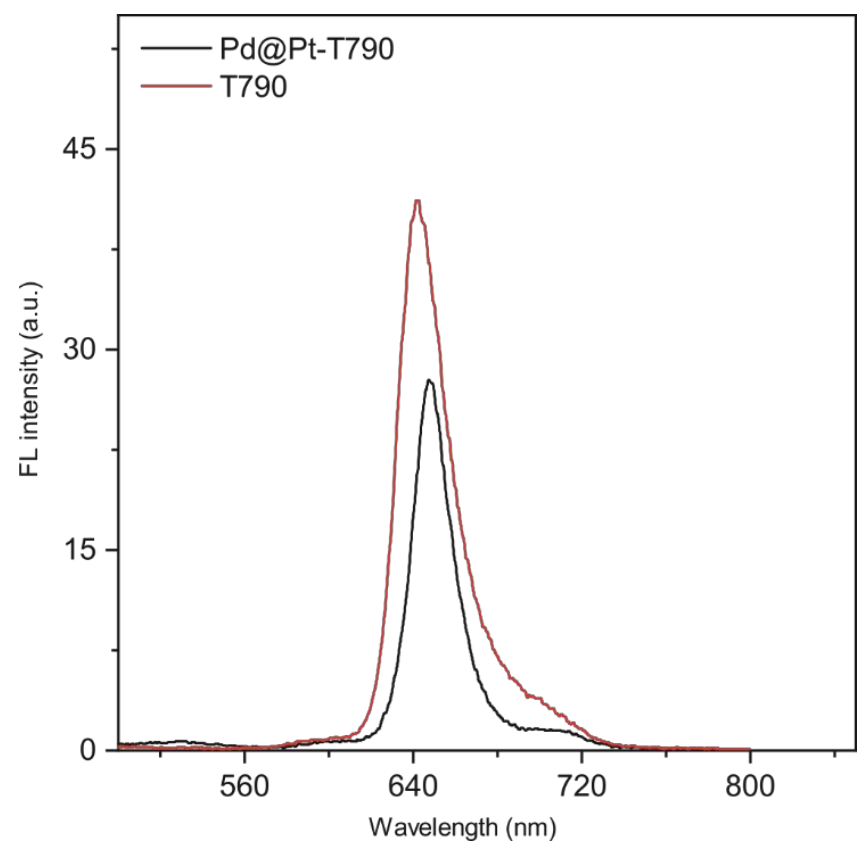

Figure S2. Fluorescence spectra of T790 and Pd@Pt-T790 in PBS. 


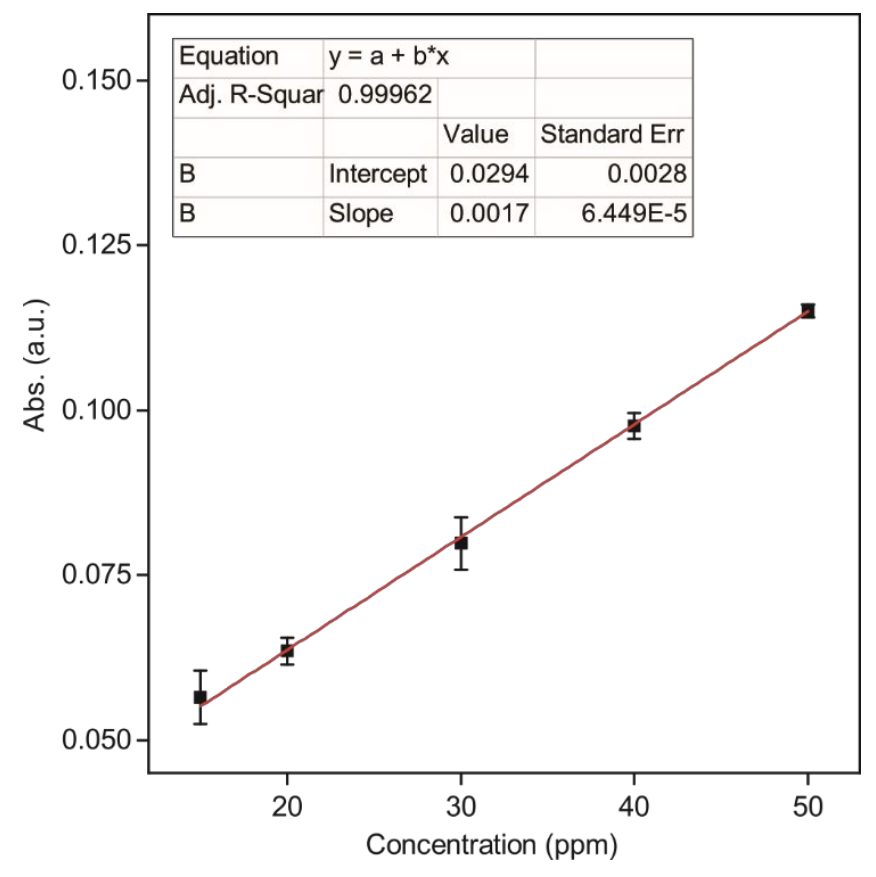

Figure S3. Standard absorption curve of T790. 


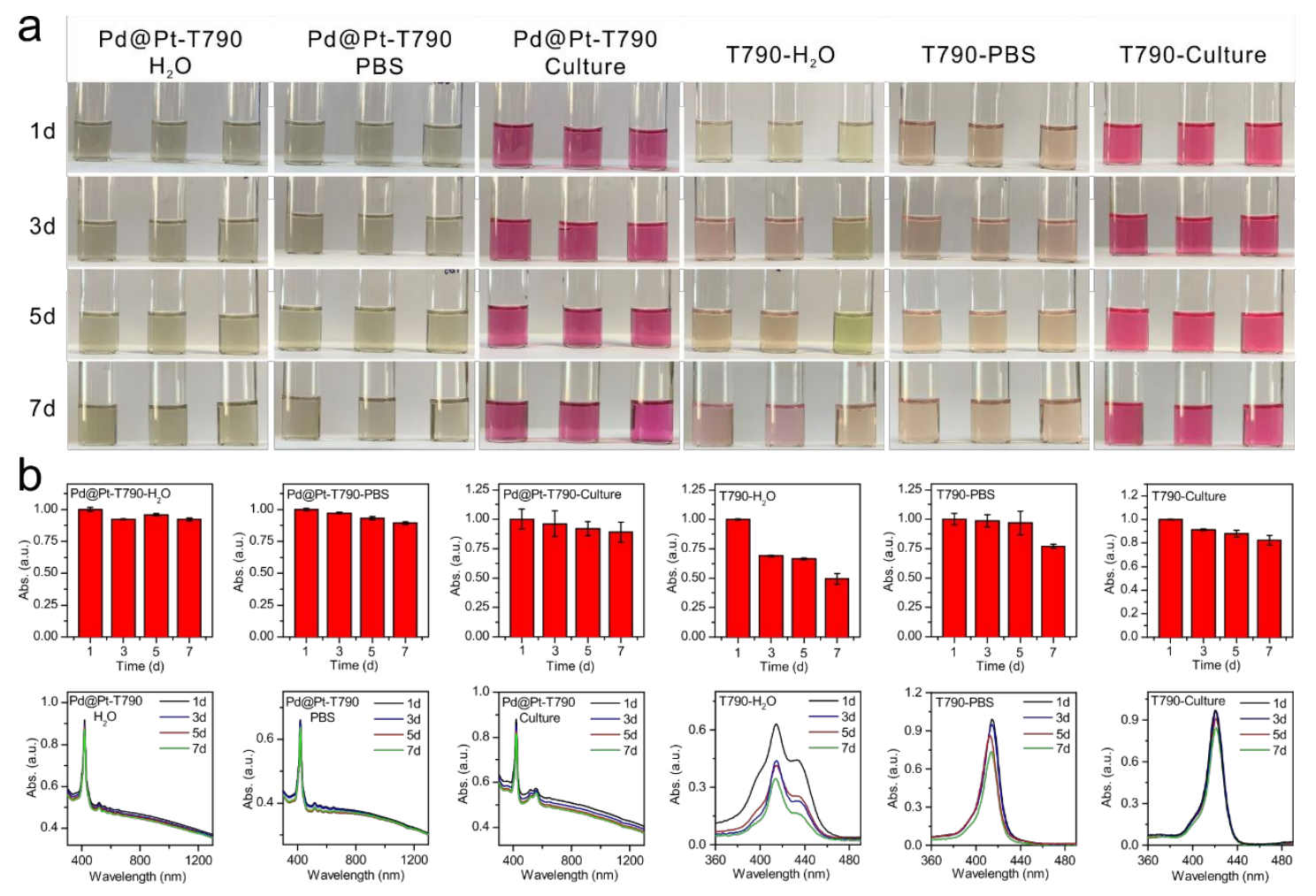

Figure S4. Dispersion stabilities of Pd@Pt-T790 and T790 in different solutions. (a) Photos of Pd@Pt-T790 and T790 dispersed in $\mathrm{H}_{2} \mathrm{O}$, PBS and culture medium within one week ( $\mathrm{n}=3$ for each group). (b) The corresponding normalized absorbances at the maximum absorption wavelength and absorption spectra of Pd@Pt-T790 and T790 in these media, respectively (The error bars represent variations among three independent measurements). 


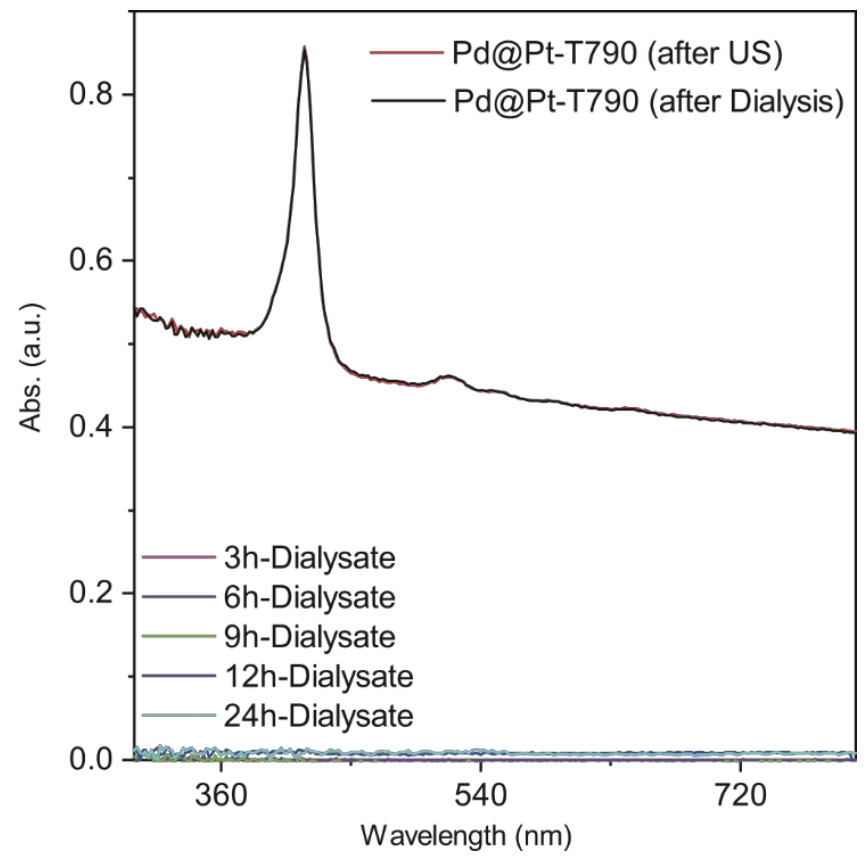

Figure S5. Dye leakage of T790 from Pd@Pt-T790 upon US irradiation (1 MHz, 0.97 W/cm², 8 min). The Pd@Pt-T790 was first subjected to US irradiation, then dialyzed for different times.
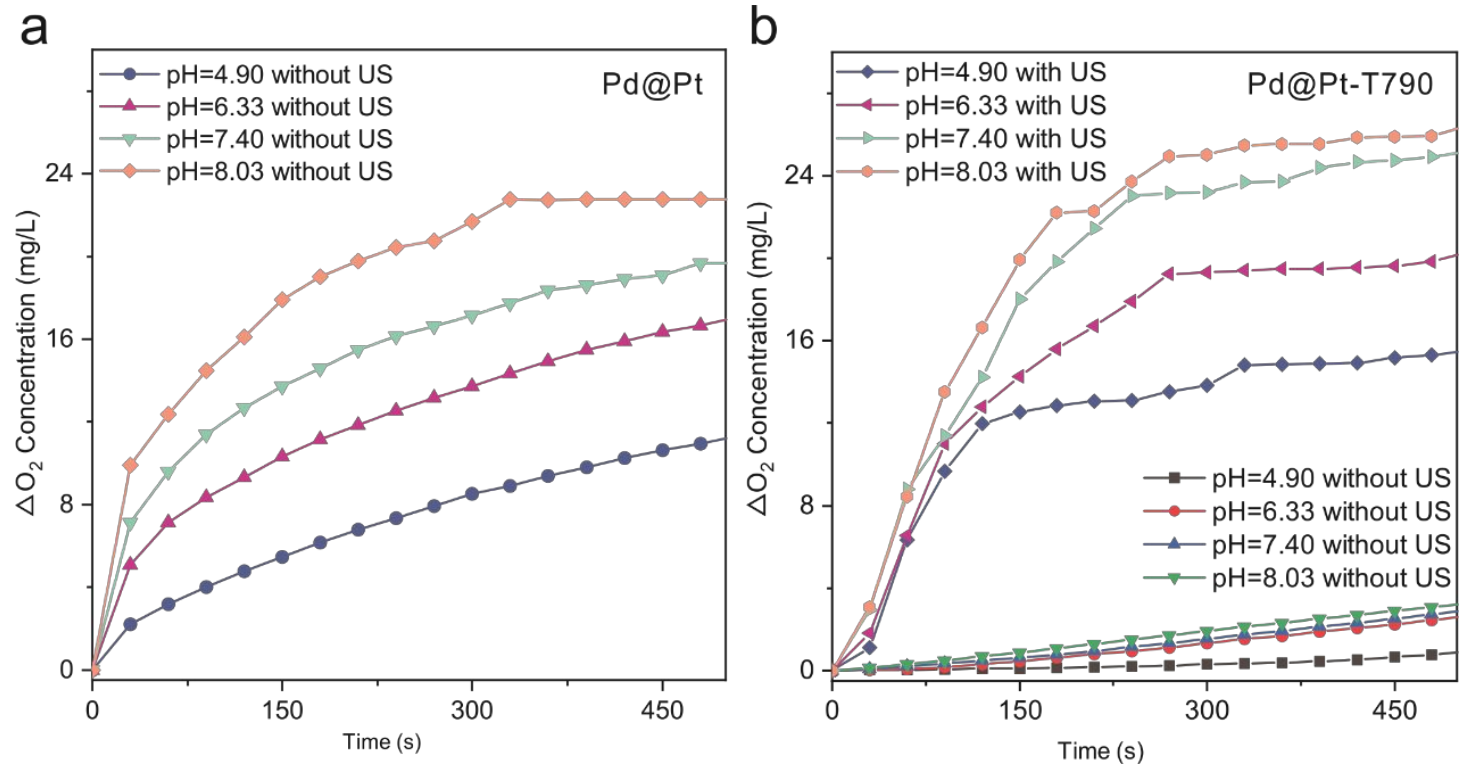

Figure S6. The influence of $\mathrm{pH}$ value on the catalase-like activity of $\operatorname{Pd} @ \operatorname{Pt}$ (a) and

Pd@Pt-T790 (b). The concentration of $\mathrm{H}_{2} \mathrm{O}_{2}$ was $1 \mathrm{mM}$. US: $1 \mathrm{MHz}, 0.97 \mathrm{~W} / \mathrm{cm}^{2}$. 

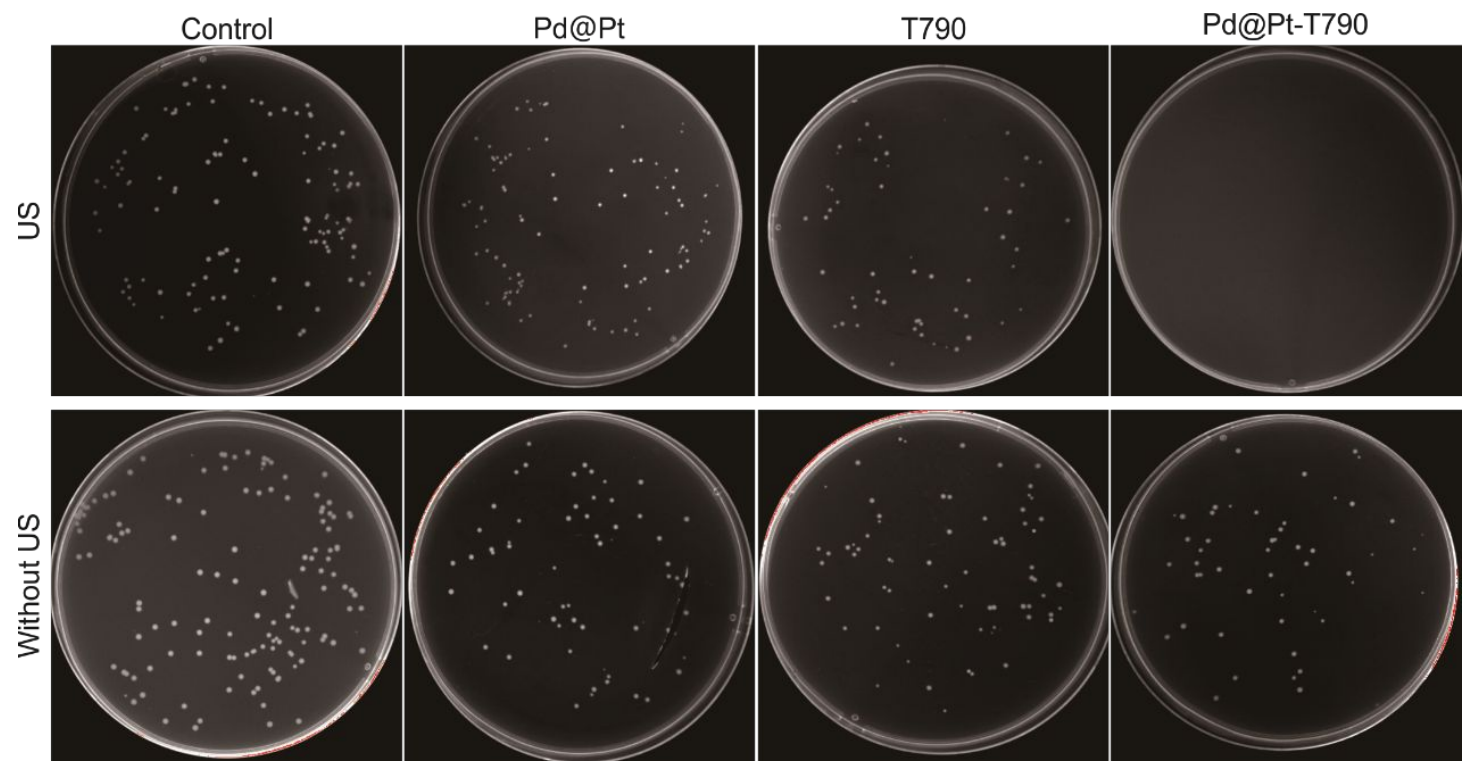

Figure S7. MRSA colony pictures after different treatments.

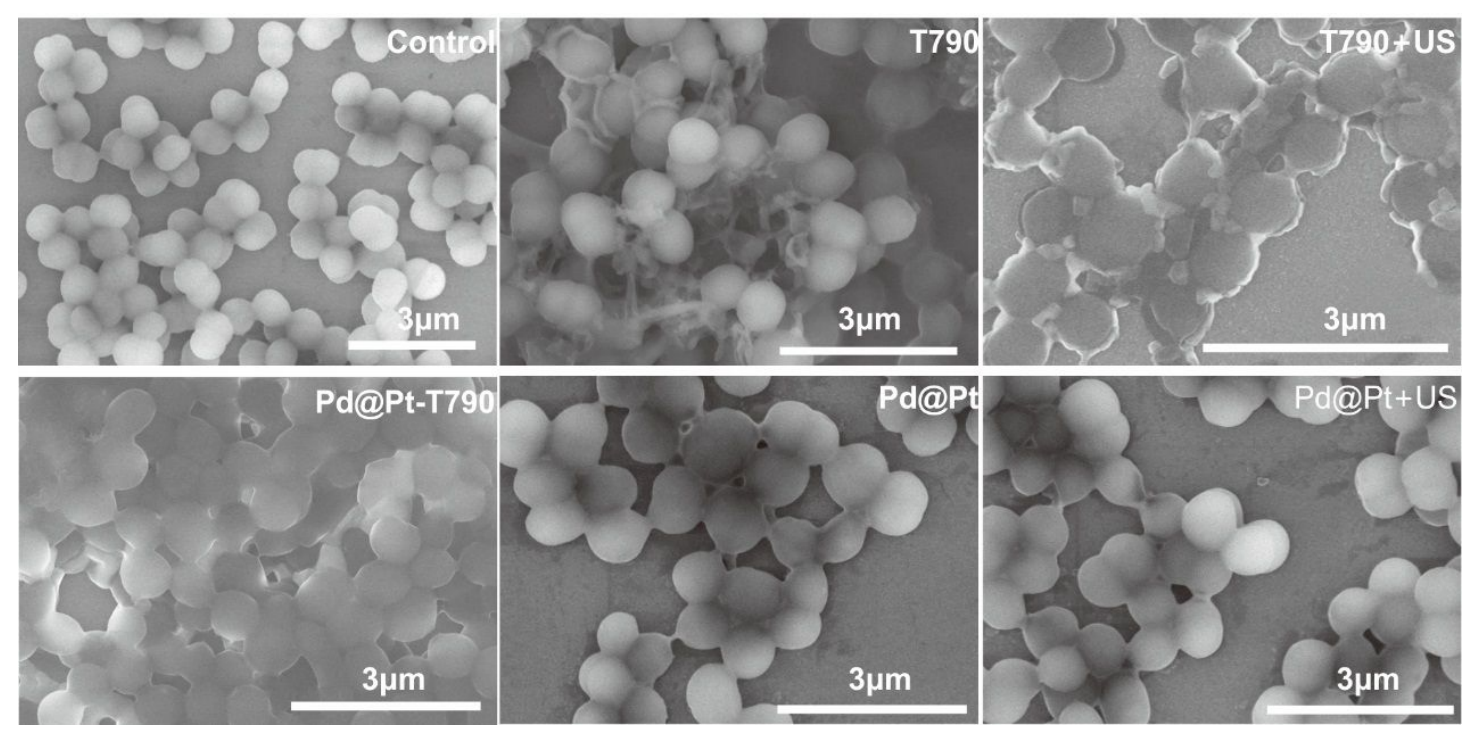

Figure S8. SEM images of MRSA after different treatments. 

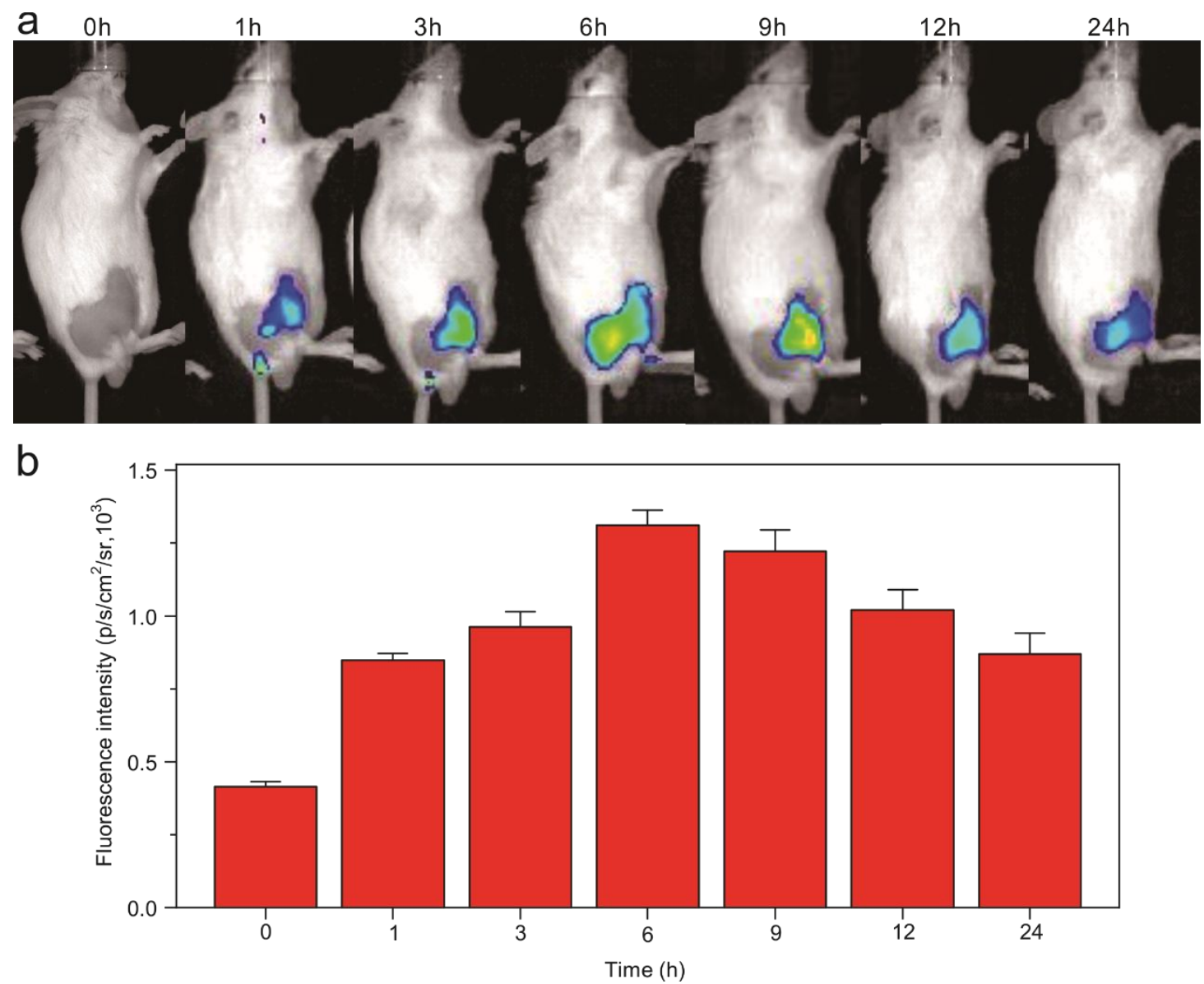

Figure S9. (a) In vivo fluorescence images of MRSA-infected mice model after tail vein injected of Pd@Pt-T790. (b) FL intensity in MRSA-infected legs.

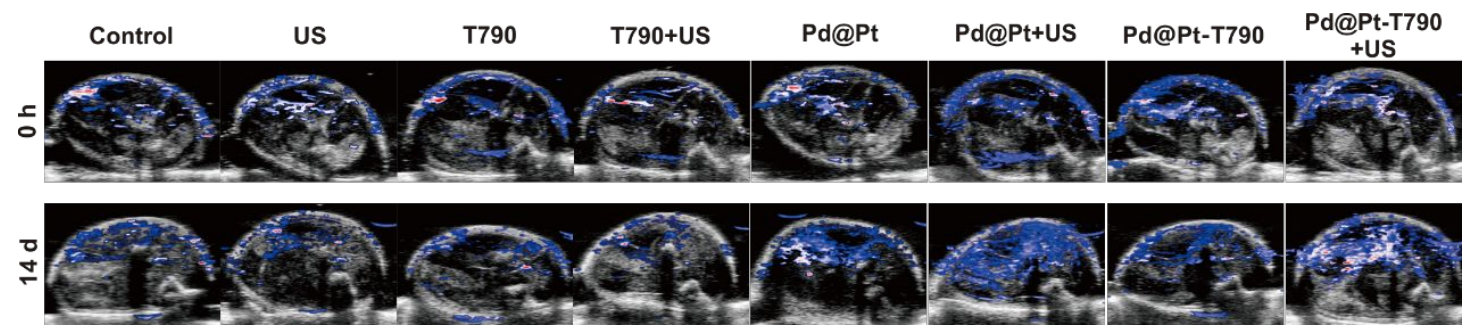

Figure S10. Representative PA images of oxyhemoglobin saturation of the MRSAinfected mice at day 14 post injection of various materials. 


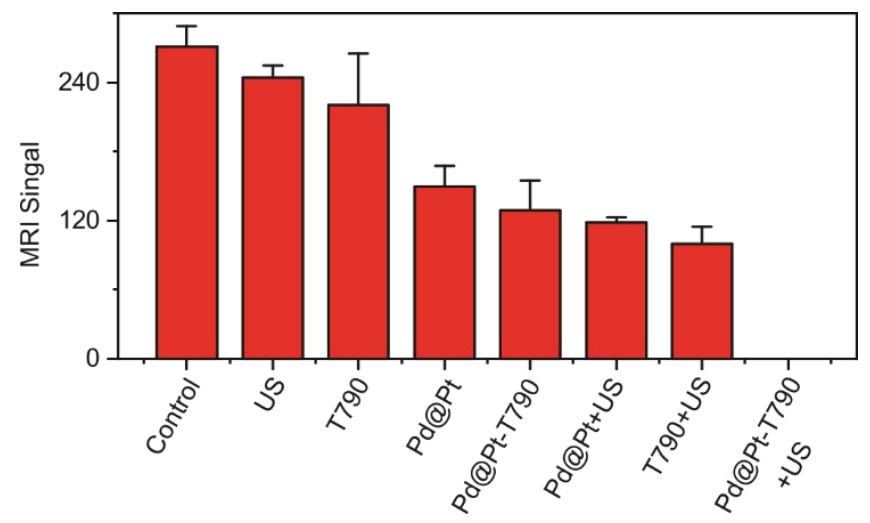

Figure S11. Quantitative results of the abscess area from MRI.

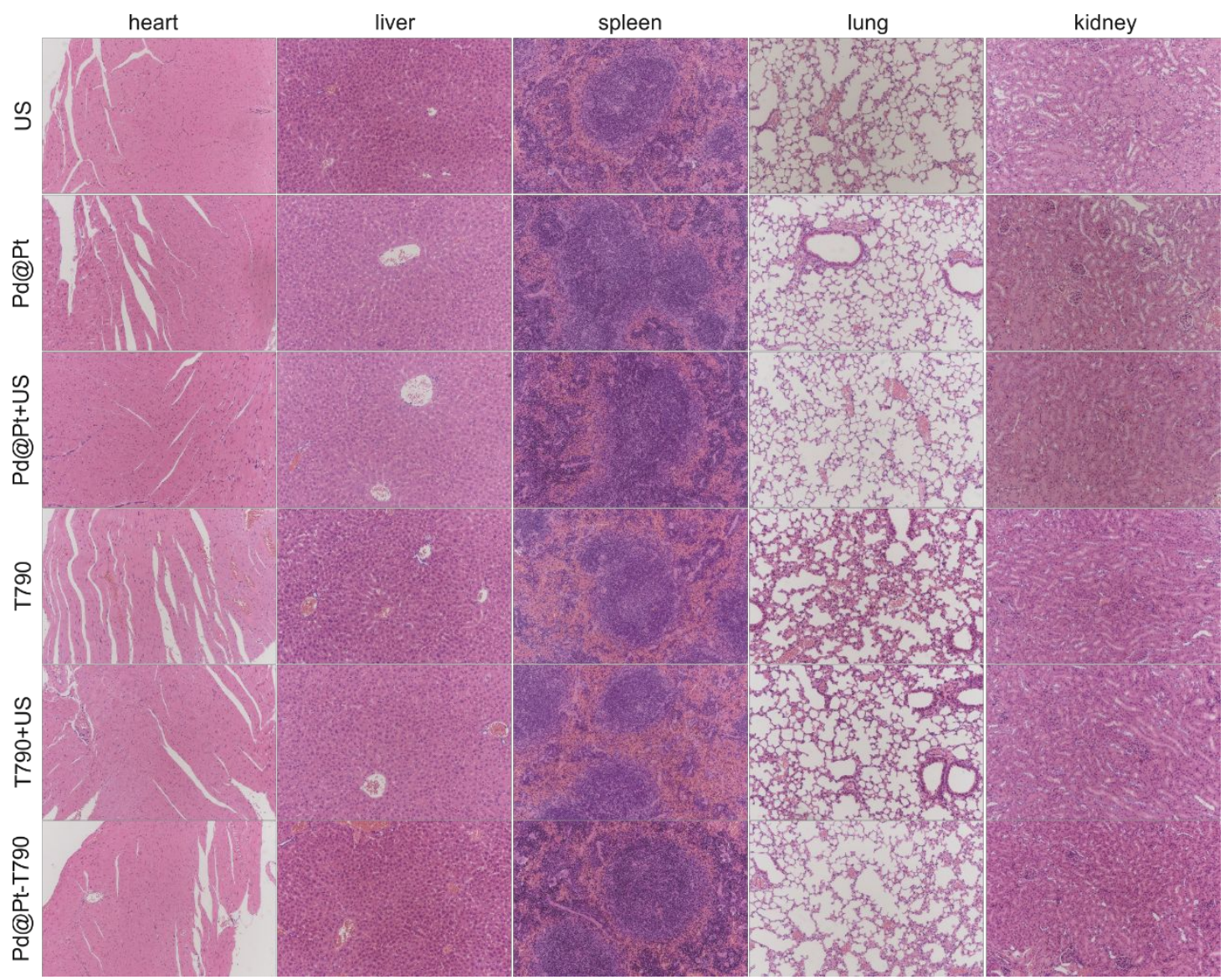

Figure S12. Histopathologic examination of the major organs including heart, liver, spleen, lung and kidney from MRSA-infected mice at day 14 after different treatments. 